\section{Fibrinólisis intravenosa en un varón de 14 años con infarto cerebral por disección de la arteria carótida interna}

Inés González-Suárez, Jorge Fernández-Travieso, Blanca Fuentes, Gerardo Ruiz-Ares, Marta Martínez-Martínez, Exuperio Díez-Tejedor

Unidad de Ictus. Departamento de Neurología. Hospital Universitario La Paz. UAM. IdiPAZ. Madrid, España.

Correspondencia: Dr. Exuperio Díez Tejedor. Unidad de Ictus. Servicio de Neurología. Hospital Universitario La Paz. IdiPAZ. Paseo de la Castellana, 261. E-28046 Madrid.

E-mail: ediez.hulp@salud.madrid.org

Aceptado tras revisión externa: 02.03.12.

Cómo citar este artículo: González-Suárez I, FernándezTravieso J, Fuentes B, Ruiz-Ares G, Martínez-Martínez M, Díez-Tejedor E. Fibrinólisis intravenosa en un varón de 14 años con infarto cerebral por disección de la arteria carótida interna. Rev Neurol 2012; 55: 189-90.

\section{(c) 2012 Revista de Neurología}

El ictus en la edad pediátrica es aquél que ocurre entre los 30 días de vida y los 18 años de edad (se excluye el período neonatal). Su incidencia es baja, y se calcula que se presenta entre 2,3 y 13 casos cada 100.000 niños. Es de etiología diversa, generalmente se encuentra asociado a múltiples factores de riesgo y las causas identificadas con mayor frecuencia son las alteraciones vasculares y las cardíacas [1].

Se asocia a altas tasas de morbimortalidad; así, se ha descrito que hasta el $10 \%$ de los pacientes fallece, presentan riesgo de recidiva del ictus en un $20 \%$ y se observan secuelas neurológicas hasta en un $70 \%$, sobre todo en forma de epilepsia secundaria o de déficits funcionales $[2,3]$. Por tanto, parece necesario aplicar un tratamiento precoz que vaya encaminado a minimizar los daños cerebrales y, con ello, las complicaciones a largo plazo.

Aunque está ampliamente establecido el beneficio del uso de la fibrinólisis intravenosa para el tratamiento de la fase aguda del ictus en adultos [4], no ocurre lo mismo en la edad pediátrica, donde la ausencia de estudios bien diseñados hace que exista una gran controversia sobre su dosificación, seguridad y beneficio.

Se presenta el caso de un varón de 14 años con diagnóstico de infarto cerebral en el territorio de la arteria cerebral media (ACM) derecha, que se trató con un factor activador del plasminógeno tisular recombinante intravenoso (rt-PA), con buenos resultados.
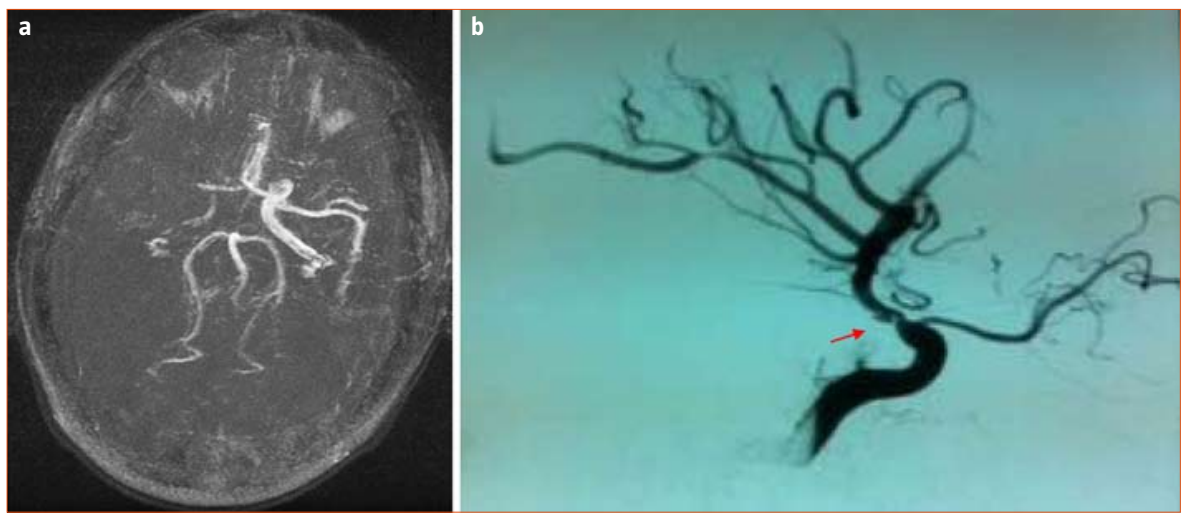

Figura. a) Angiorresonancia de arterias del polígono de Willis que demuestra obstrucción de la arteria cerebral media derecha; b) Arteriografía cerebral en la que se observan signos de disección arterial en la arteria carótida interna derecha (flecha).

Varón de 14 años de edad natural de Ecuador, con antecedentes de plaquetopenia de etiología no filiada desde los 5 años de edad, tratado mediante esplenectomía y profilaxis con penicilina desde entonces. Acude a urgencias por presentar cefalea hemicraneal derecha asociada a hemiparesia izquierda transitoria autolimitada a 15 minutos, en el contexto de una competición de natación. Tras la valoración en urgencias, en la que el paciente se encuentra asintomático y cuya una tomografía computarizada (TC) craneal resulta normal, se decide darle el alta hospitalaria, con posterior estudio ambulatorio.

Cuatro días después regresa a urgencias por una disminución de fuerza en el hemicuerpo izquierdo y cefalea intensa de hora y media de evolución. En la exploración clínica general no se objetivaron alteraciones. Desde el punto de vista neurológico, presenta hemianopsia homónima izquierda, parálisis facial central izquierda, hemiparesia izquierda y hemihipoestesia táctil y algésica de ese hemicuerpo, así como extinción visual y sensitiva izquierda, con una puntuación en la escala NIHSS al ingreso de 12. Se realizaron análisis de sangre, radiografía de tórax y electrocardiograma, los cuales no presentaron alteraciones. Se solicitó una resonancia magnética (RM) con angio-RM cerebral urgente, en la que se observaron alteraciones compatibles con infarto cerebral en el territorio de la ACM derecha, con oclusión de la ACM derecha desde $\mathrm{M} 1$ y caída del flujo en la arteria carótida interna $(\mathrm{ACl})$ derecha.

Al no existir contraindicaciones, y tras la firma del consentimiento informado por la familia, se llevó a cabo fibrinólisis intravenosa con
rt-PA dentro del protocolo de uso compasivo por edad menor a 18 años. Durante la infusión se monitorizó la ACM derecha mediante Doppler transcraneal, y, tras comprobar la ausencia de mejoría clínica a los 30 minutos, así como la persistencia de la obstrucción arterial, se decidió realizar un tratamiento de recanalización mediante intervencionismo intravascular. La arteriografía diagnóstica constató la recanalización de la ACM derecha, en la que se observaron signos de disección arterial en la $\mathrm{ACl}$ derecha intracraneal (Figura). Neurológicamente, el paciente evolucionó de forma favorable, con una puntuación en la escala NIHSS a las 6 y 24 horas de 3 y 0 puntos, respectivamente. La TC craneal efectuada a las 24 horas descartó la presencia de complicaciones hemorrágicas.

El paciente fue dado de alta asintomático, y se le recomendó un antiagregante plaquetario como prevención secundaria con ácido acetilsalicílico $300 \mathrm{mg} /$ día.

El ictus en la edad pediátrica es una enfermedad poco frecuente que difiere en gran medida del acaecido en el adulto. En la mitad de los niños se identifica un factor de riesgo previo al ictus y, en casi dos tercios, se descubre más de un factor de riesgo durante el cribado diagnóstico [1]. Los factores identificados con mayor frecuencia como causa de éste son la arteriopatía, seguido de las infecciones (meningitis o sepsis) y las anomalías cardíacas (congénitas o adquiridas). Sin embargo, la etiología del ictus no se diagnostica hasta casi en el $30 \%$ de los niños [5]. La clínica variará según la edad y la localización de la lesión; así, en menores de un año, 
suele manifestarse como una encefalopatía con disminución del nivel de conciencia, mientras que en niños mayores, el inicio de un déficit neurológico focal de instauración brusca es lo más característico.

El tratamiento del ictus agudo en pacientes pediátricos no está bien establecido; recientemente, se han publicado varias guías específicas $[1,6-8]$, aunque el uso de la terapia fibrinolítica intravenosa sigue siendo un tema de controversia. Puede extrapolarse que el beneficio demostrado en adultos sugiere un efecto similar en niños; sin embargo, esta afirmación no es del todo correcta, ya que hay diferencias sustanciales entre ambos grupos de edades. En primer lugar, la etiología del ictus en adultos y en niños es diferente; en niños son frecuentes las anomalías cardíacas y los estados de hipercoagulabilidad como causa subyacente, de modo que estos trombos son frescos en fibrina $y$, por tanto, más sensibles a la lisis con rt-PA [9]. En segundo lugar, el sistema de coagulación en niños no ha madurado completamente, así que, hasta los 16 años, los niveles del factor activador del plasminógeno (t-PA) son un $50 \%$ menores que los del adulto; por otro lado, los valores del factor activador del inhibidor del plasminógeno 1 (PAl-1) son mayores en niños. Ambos hechos, que sugieren un sistema fibrinolítico inmaduro en niños, hacen sospechar que se precisen dosis mayores de t-PA para conseguir el efecto fibrinolítico deseado [10]. Por último, en niños, existe una gran demora diagnóstica, y es frecuente diagnosticar al paciente pasadas las 4,5 horas recomendadas para el uso del tratamiento. Todo esto hace que en las guías de manejo del ictus agudo en niños no esté establecido su uso de manera generalizada, recomendando la individualización de la indicación terapéutica según el caso. Por otro lado, se han publicado diversos casos sobre el empleo de la fibrinólisis en niños con resultados excelentes y efectos secundarios mínimos [11-16], que han mostrado el beneficio y la seguridad del uso del rt-PA en la edad pediátrica.

Aun así, serían necesarios ensayos bien diseñados para establecer la correcta dosificación, indicación y perfil de seguridad del tratamiento trombolítico intravenoso en el infarto cerebral en niños.

Bibliografía

1. Roach ES, Golomb MR, Adams R, Biller J, Daniels $S$, DeVeber $G$, et al. Management of stroke in infants and children. A scientific statement from a special group of the American Heart Association Stroke Council and the Council on Cardiovascular Disease in the Young. Stroke 2008; 39: 2644-91.

2. Amlie-Lefond A, Sébire G, Fullerton HJ. Recent developments in childhood arterial ischaemic stroke. Lancet Neurol 2008; 7: 425-35.

3. DeVeber G, MacGregor D, Curtis R, Mayank S. Neurologic outcomes in survivors of childhood arterial ischemic stroke and sinovenous thrombosis. J Child Neurol 2000; 5: 316-24.

4. Adams HP Jr, Del Zoppo G, Alberts MJ, Bhatt DL, Brass L, Furlan A, et al. Guidelines for the early management of adults with ischemic stroke: a guideline from the American Heart Association/American Stroke Association Stroke Council, Clinical Cardiology Council, Cardiovascular Radiology and Intervention Council, and the Atherosclerotic Peripheral Vascular Disease and Quality of Care Outcomes in Research Interdisciplinary Working Groups: the American Academy of Neurology affirms the value of this guideline as an educational tool for neurologists. Circulation 2007; 115: e478-534.

5. Fullerton HJ, Wu YW, Sidney S, Johnston SC. Risk of recurrent childhood arterial ischemic stroke in a population-based cohort: the importance of cerebrovascular imaging. Pediatrics 2007; 119: 495-501.

6. Paediatric Stroke Working Group. Stroke in childhood: clinical guidelines for diagnosis, management and rehabilitation. London: Royal College of Physicians of London; 2004.

7. Monagle P, Chan A, Massicotte P, Chalmers E, Michelson AD. Antithrombotic therapy in children: the Seventh ACCP Conference on Antithrombotic and Thrombolytic Therapy. Chest 2004; 126 (Suppl 3): S645-87.

8. Janjua N, Nasar A, Lynch JK, Qureshi Al. Thrombolysis for ischemic stroke in children: data from the nationwide inpatient sample. Stroke 2007; 38: 1850-4.

9. Belvís R. Thrombolysis for acute stroke in pediatrics. Stroke 2007; 38: 1722-3.

10. Amlie-Lefond C, Chan AK, Kirton A, DeVeber G, Hovinga CA, Ichord R, et al. Thrombolysis in acute childhood stroke: designs and challenges of the thrombolysis in pediatric stroke clinical trial. Neuroepidemiology 2009; 32: 279-86.

11. Thirumalai SS, Shubin RA. Successful treatment for stroke in a child using recombinant tissue plasminogen activator. J Child Neurol 2000; 15: 558.

12. Noser EA, Felberg RA, Alexandrov AV. Thrombolytic therapy in an adolescent ischemic stroke. J Child Neurol 2001; 16: 286-8.

13. Carlson MD, Leber S, Deveikis J, Silverstein FS. Successful use of rt-PA in pediatric stroke. Neurology 2001; 57: 157-8.

14. Shuayto MI, Lopez JI, Greiner F. Administration of intravenous tissue plasminogen activator in a pediatric patient with acute ischemic stroke. J Child Neurol 2006; 21: 604-6.

15. Arnold M, Steinlin M, Baumann A, Nedeltchev K, Remonda L, Moser JL, et al. Thrombolysis in childhood stroke: report of two cases and review of the literature. Stroke 2009; 40: 801-7.

16. Amlie-Lefond C, DeVeber G, Chan AK, Benedict S, Bernard T, Carpenter J, et al. Use of alteplase in childhood arterial ischaemic stroke: a multicentre, observational, cohort study. Lancet Neurol 2009; 8: 530-6.
Hepatitis autoinmune en un paciente con esclerosis múltiple en tratamiento con acetato de glatiramero

Maialen Arruti a Tamara Castillo-Triviño a,b,c, Patricia de la Riva a , José Félix Martí-Massóa,c, Adolfo López de Munain a,c, Javier Olascoaga a,b,c

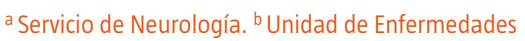
Desmielinizantes. Hospital Universitario Donostia.

cÁrea de Neurociencias. Instituto Biodonostia. San Sebastián, Guipúzcoa, España.

Correspondencia: Dra. Maialen Arruti González.

Servicio de Neurología. Hospital Universitario Donostia. P.o Doctor Begiristain, 107. E-20014 Donostia-San Sebastián (Guipúzcoa).

E-mail:m_arruti@hotmail.com

Aceptado tras revisión externa: 25.05.12.

Cómo citar este artículo: Arruti M, Castillo-Triviño T, De la Riva P, Martí-Massó JF, López de Munain A, Olascoaga J. Hepatitis autoinmune en un paciente con esclerosis múltiple en tratamiento con acetato de glatiramero. Rev Neurol 2012; 55: 190-2.

(C) 2012 Revista de Neurología

El acetato de glatiramero es un copolímero sintético de aminoácidos análogo a la proteína básica de la mielina. Es uno de los fármacos inmunomoduladores más empleados en el tratamiento modificador de la historia natural de la esclerosis múltiple. Está indicado como fármaco de primera línea en el síndrome clínico aislado o síndrome desmielinizante aislado y en formas de esclerosis múltiple remitente recurrente (EMRR). También se utiliza como alternativa al interferón- $\beta$ en pacientes con EMRR y respuesta subóptima, efectos adversos o intolerancia a dicha medicación [1]. Es un fármaco con buena tolerancia y baja proporción de efectos adversos 0 abandonos de tratamiento. La hepatitis aguda es un efecto secundario muy poco frecuente.

Mujer de 46 años de edad, diagnosticada de EMRR desde hace siete años y que había recibido tratamiento con interferón- $\beta$ durante seis años, en los que permaneció libre de brotes, sin discapacidad (EDSS $=1$ ) y sin efectos adversos llamativos. Tras presentar varios brotes consecutivos que originaron un claro empeoramiento clínico (EDSS $=4,5$ ) y que precisaron tratamiento con pulsos de corticoides intravenosos, se suspendió el tratamiento con interferón- $\beta$ y se inició el tratamiento con acetato de glatiramero.

A los dos meses de iniciar dicho tratamiento, la paciente acudió a la consulta por malestar general. En la exploración, presentaba icte- 